\title{
Results of the ESO-SEST key programme: CO in the Magellanic Clouds
}

\section{The 30 Doradus Complex}

\author{
M.L. Kutner ${ }^{1,2}$, M. Rubio ${ }^{3}$, R.S. Booth ${ }^{4}$, F. Boulanger ${ }^{5,6}$, Th. de Graauw ${ }^{7}$, G. Garay ${ }^{3}$, F.P. Israel , $^{8}$ \\ L.E.B. Johansson ${ }^{4}$, J. Lequeux ${ }^{5,9}$, and L.-Å. Nyman $^{10}$ \\ 1 NRAO, 949 N. Cherry Av., Campus Building 65, Tucson, Arizona 85721-0655, U.S.A. \\ 2 Department of Physics, Rensselaer Polytechnic Institute, Troy, NY 12180, U.S.A. \\ 3 Departamento de Astronomía, Universidad de Chile, Casilla 36-D, Santiago, Chile \\ 4 Onsala Space Observatory, S-43900 Onsala, Sweden \\ 5 Radioastronomie, Ecole Normale Superieure, 24 Rue Lhomond, 75231 Paris Cedex 05, France \\ 6 Institut d'Astrophysique Spatiale, Bat. 120, Université de Paris-XI, 91045 Orsay Cedex, France \\ 7 Laboratorium voor Ruimteonderzoek, SRON, Postbus 800, NL-9700 AV Groningen, The Netherlands \\ 8 Sterrewacht, Postbus 9513, NL-2300 Leiden, The Netherlands \\ 9 DEMIRM, Observatoire de Paris, 61 Av. de l'Observatoire, 75014 Paris, France \\ 10 European Southern Observatory, Casilla 19001, Santiago 19, Chile
}

Received April 25; accepted July 7, 1996

\begin{abstract}
We report observations of ${ }^{12} \mathrm{CO}(1 \rightarrow 0)$ emission from molecular clouds in a complex extending $\sim 2 \mathrm{kpc}$ south of 30 Dor in the Large Magellanic Cloud. The observations were taken on the Swedish-ESO Submillimetre Telescope (SEST) as part of the Key Programme: CO in the Magellanic Clouds. This paper presents observations of the emission from the Central and Southern parts of that complex. In both parts, the strongest emission has $T_{\mathrm{A}}^{*} \sim 4 \mathrm{~K}$. This is a factor of $\sim 3$ to 5 weaker than we would expect for Milky Way GMCs observed at a distance of $50 \mathrm{kpc}$. In the Central region, the emission is dominated by a $600 \mathrm{pc}$ long, slightly curved feature, which is clearly seen in peak and integrated intensity maps. When we look at this emission in $5 \mathrm{~km} \mathrm{~s}^{-1}$ velocity ranges, it breaks into 22 clouds whose sizes and line profiles look like that from Milky Way molecular clouds. The Southern region is clearly separated from the Central region, and its emission can be broken into 5 clouds.
\end{abstract}

Key words: Galaxy: Magellanic Clouds — interstellar medium: molecules — interstellar medium: clouds interstellar medium: molecules, millimeter lines radio lines: molecular

Send offprint requests to: M.L. Kutner

* based on results collected at the European Southern Observatory, La Silla, Chile.

\section{Introduction}

The Magellanic Clouds provide unique opportunities for studying molecular clouds and star formation in galaxies whose environment is very different from that of the Milky Way. At the $50 \mathrm{kpc}$ distance of the Large Magellanic Cloud (LMC), 1 arc minute covers 15 pc. This means that we can make $\mathrm{CO}$ maps of individual molecular clouds with even modest single dishes. For example, the 15-m SwedishESO Submillimetre Telescope (SEST) provides an angular resolution of $43^{\prime \prime}$ at $2.6 \mathrm{~mm}$, corresponding to a linear resolution of $10 \mathrm{pc}$. This is well suited to measure the properties of clouds that are a few tens of pc in extent. In addition, the $J=2 \rightarrow 1$ line can provide $5 \mathrm{pc}$ resolution where necessary. Prior to the availability of the SEST, information was obtained on the extent of CO emission in the LMC (Cohen et al. 1988) and in the SMC (Rubio et al. 1991) using a $1 \mathrm{~m}$ telescope.

The problem of star formation in irregular galaxies is very different from that in spirals. In irregulars there is no organized pattern of star formation. Rather, there appear to be isolated bursts. It is interesting to know what triggers those bursts, and whether there is some propagation of the star formation activity. Also, the interstellar medium in irregulars is dominated by atomic hydrogen, rather than molecular hydrogen. However, in the Milky Way (and other spirals) we have come to accept the idea that molecular clouds are necessary for star formation. Therefore, the role of HI in star formation could be 
potentially more important in irregulars than in the Milky Way. Also, understanding star formation in the HI dominated, low metallicity, environment of these galaxies, might provide clues on the formation of the first stellar generations in all galaxies.

To study molecular clouds, we rely on millimeter wave observations of carbon monoxide $(\mathrm{CO})$, a trace constituent. In the Milky Way, the issue of how we convert from the $\mathrm{CO}$ luminosities of molecular clouds to their masses has been a topic of considerable debate. (See e.g. Combes 1991, and references therein for some discussion of this problem). Even though there is a growing consensus among observers on how to treat this problem in the Milky Way, the question of how to approach it in other galaxies is still unresolved. A major source of uncertainty is the effect of metallicity and gas-to-dust ratio on the conversion factor. In the LMC, the metallicity is a factor of four lower than in our Galaxy (Dufour 1984) and the gas to dust ratio is four times the Galactic value (Koornneef 1982). Moreover, in the Milky Way, the consensus has emerged after studies that included full maps of a statistically significant sample of clouds. In the Magellanic Clouds, we have the opportunity to make such maps, even with the modest angular resolution of single dishes.

The study of $\mathrm{CO}$ emission has been designated as a Key Programme on the SEST. As such, approximately 40 half days per year have been devoted to observations since 1988 May . The goal of the Key Programme is to produce fully sampled maps of $\mathrm{CO}$ emission from several representative areas in the LMC and SMC. With resolution and sensitivity to map many types of regions, we can address a number of important questions:

1. What is the nature of the molecular emission from the Magellanic Clouds? Does the emission come mostly from well defined clouds, as in the Milky Way, or is there extensive low level emission?

2. If there are well defined clouds, what are their size distributions? Is most of emission coming from a relatively small number of giant molecular clouds (GMCs), with extents of tens of parsecs, as in the Milky Way, or is there a higher proportion of smaller clouds? Different theories of cloud formation, evolution and destruction predict different size distributions.

3. What are the cloud masses? We can determine virial masses for a statistically significant sample of clouds. There is growing evidence that the virial mass is a good indicator of cloud mass (to within a factor of two) for large ensembles of clouds.

4. We can address the question of the conversion from $\mathrm{CO}$ luminosity, $L_{\mathrm{CO}}$ to $\mathrm{H}_{2}$ mass. In particular by comparing that conversion from the Milky Way to the LMC to the SMC, we can see how changing metallicity affects that conversion.

5. Once we have a good idea of the distribution of molecular material we can also address the questions of star formation activity and morphology. Of course, these require infrared and radio observations, that are beyond the immediate goals of the Key Programme.

Some of the initial results are presented in Johansson (1991) and Rubio (1991). The results of selected pointed observations in the LMC are presented by Israel et al. (1990; hereafter Paper I). Observations of the SMC are reported by Rubio et al. (1993a,b, hereafter Paper II and Paper III, Lequeux et al. 1994 Paper IV, and Rubio et al. 1996 Paper V).

The regions chosen for extensive mapping are shown in Fig. 1, superimposed on the contours of $\mathrm{CO}$ emission mapped by Cohen et al. (1988) with an 8.8 arcminute beam. Three regions have been singled out, (1) the immediate vicinity of the HII region associated with 30 Dor, (2) the clouds in the vicinity of the isolated HII region N11, (3) the dark cloud complex extending some $2 \mathrm{kpc}$ south of 30 Dor. It is this third region that is the subject of this Paper. Extensive CO emission associated with this feature was noted by Cohen et al. (1988). The SEST ${ }^{12} \mathrm{CO}(1 \rightarrow 0)$ maps are presented in this Paper, and the individual cloud properties are analyzed in Paper VII.

These regions were selected as result of a fully sampled unbiased single strip extending for $3.5^{\circ} \mathrm{N}-\mathrm{S}$ through the center of the 30 Doradus Complex. Part of that strip is shown in Fig. 1. The purpose of observing this strip was in recognition of the fact that there is a large jump in resolution from the Cohen et al. data to the SEST data. If the emission is patchy, then regions inside the lowest contour in the Cohen et al. map might have no emission as seen from the SEST, and regions outside the lowest contour might have detectable emission. After the full strip was made, some regions where emission was detected were chosen for full mapping. In this sense, the data in this paper are different than for Paper I, in which IRAS peaks were selected for study. The data presented in this Paper fall into two distinct regions in Fig. 1, and we designate them as the Central and Southern regions of the 30 Dor Complex.

\section{Observations and reductions}

The observations of the ${ }^{12} \mathrm{CO}(1 \rightarrow 0)$ line were made in 1989 May, June and October, 1990 January July, and 1991 May, using the SEST, located on La Silla, Chile, at an altitude of $2200 \mathrm{~m}$. At $2.6 \mathrm{~mm}$ the antenna HPBW is $43^{\prime \prime}$.

The receiver was a single channel cooled Schottky mixer, tuned to be optimized as a single sideband receiver. The effective system temperature on the sky, $T_{\mathrm{SYS}}^{*}$ (corrected for rearward spillover and atmospheric attenuation) was typically $\sim 500 \mathrm{~K}$. Spectral resolution was provided by a 2000 channel AOS with a resolution of $43 \mathrm{kHz} /$ channel. Observations were made in a frequency switching mode, with a throw of 15 or $20 \mathrm{MHz}$, depending on the velocity range over which emission was seen. The line was always in the spectrometer bandpass for both halves of the switching cycle. The $15 \mathrm{MHz}$ shift for frequency switching 


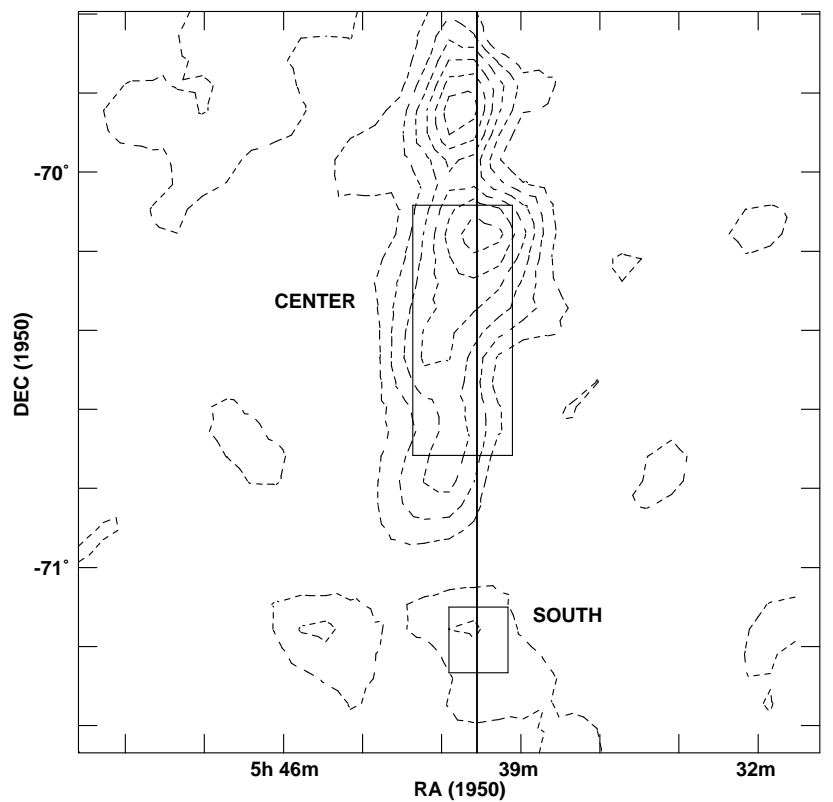

Fig. 1. Locations of regions observed with the SEST, superimposed on the $\mathrm{CO}$ contours of the data taken from Cohen et al. (1988). The regions studied with the SEST are shown as rectangular outlines and part of the north-south strip, observed at $20^{\prime \prime}$ intervals, is marked with a vertical line

corresponds to $39 \mathrm{~km} \mathrm{~s}^{-1}$, so we could have seen emission approximately $30 \mathrm{~km} \mathrm{~s}^{-1}$ on either side of the center velocity. Spectra were folded in the final processing to improve the rms by $\sqrt{2}$. Most of the spectra required the removal of third order baselines in the final processing. This could be done reliably because the lines are relatively narrow, and we have a large number of channels to determine the baseline accurately. For the reduction of the data, groups of five adjacent channels were averaged to provide an effective resolution of $215 \mathrm{kHz}$, corresponding to a velocity resolution of $0.55 \mathrm{~km} \mathrm{~s}^{-1}$.

Intensity calibration was performed using the chopper technique, in which the receiver alternately looks at the sky and an ambient temperature absorber during the calibration phase. The time between calibrations was typically 10 to 30 minutes, depending on elevation and sky stability. The intensities are reported as $T_{\mathrm{A}}^{*}$ (Kutner \& Ulich 1981), in which the intensities are corrected for atmospheric attenuation and rearward spillover. To convert to $T_{\mathrm{R}}^{*}$, one must divide $T_{\mathrm{A}}^{*}$ by the forward spillover and scattering efficiency, $\eta_{\text {FSS }}=0.92$. This value was determined from observations of the Moon. To convert to $T_{\mathrm{MB}}$ the brightness temperature for a source that uniformly fills the main beam, one must divide $T_{\mathrm{A}}^{*}$ by the product of $\eta_{\text {FSS }}$ and source coupling efficiency for an object that uniformly fills the main beam, $\eta_{\mathrm{C}}(\mathrm{MB})=0.80$ for the SEST. This means that $T_{\mathrm{MB}}=T_{\mathrm{A}}^{*} / 0.74$. Unless otherwise stated, contour maps and spectra are presented on the $T_{\mathrm{A}}^{*}$ scale. Tabulated properties, such as peak temperature or integrated intensity, as well as derived quantities, such as CO luminosities, will be presented on the $T_{\mathrm{MB}}$ scale, since we are talking about objects for which there is some idea of the extent relative to the beam size. To convert those values back to $T_{\mathrm{A}}^{*}$ they should be multiplied by 0.74 , and convert back to $T_{\mathrm{R}}^{*}$ they should be multiplied by 0.80 .

Pointing was checked periodically on the $\mathrm{SiO}$ maser, $\mathrm{R}$ Dor, which is near the LMC in the sky. This allowed pointing checks in the same azimuth and elevation range as the source. The rms pointing errors were typically $4^{\prime \prime}$ in each axis.

The general observing philosophy for the Key Programme was to produce fully sampled maps. For ${ }^{12} \mathrm{CO}(1 \rightarrow 0)$, this means a grid spacing of $20 "$ (or 5 pc at the LMC distance). Integration times were adjusted to provide an rms noise level in the final (folded and frequency degraded) spectra of $0.10 \mathrm{~K}$ (on the $T_{\mathrm{A}}^{*}$ scale). Typical integration time per map position were approximately 4 minutes under the best conditions.

As mentioned above, the regions studied here were chosen on the basis of fully sampled observations of a strip, partially shown in Fig. 1. That strip is at $\alpha(1950)=$ $5^{\mathrm{h}} 40^{\mathrm{m}} 30^{\mathrm{s}}$ with $\delta(1950)$ ranging from $-68^{\circ} 45^{\prime} 00^{\prime \prime}$ to $-72^{\circ} 00^{\prime} 00^{\prime \prime}$. Of the 585 positions in the strip, emission above our threshold was found in 88 ( $\sim 15 \%$ of the) positions. The detections were also concentrated in a way that suggested that the strip was cutting through clouds with large gaps in between. The clouds thus found in the strip were the ones selected for detailed mapping.

\section{Results}

In Fig. 2, we show several sample spectra from both the Central and Southern regions. The RA and DEC offsets are with respect to $\alpha(1950)=5^{\mathrm{h}} 40^{\mathrm{m}} 30^{\mathrm{s}}, \delta(1950)=$ $-69^{\circ} 52^{\prime} 00^{\prime \prime}$. These spectra provide an idea of the quality of the data. In addition, we can see how the line falls off from the cloud center to edge. Also, we get an idea of how the line profiles change as one moves across the cloud. The general appearance of the line profiles is similar to that seen in Milky Way molecular clouds, especially those seen in the outer Galaxy, where there is little confusion along the line of sight (Mead \& Kutner 1988). The major difference is that the LMC lines are much weaker than the Galactic counterparts.

Contour maps of the overall $\mathrm{CO}$ emission are shown in Fig. 3. In Fig. 3a (Central region) and 3c (Southern region) we present contour maps of peak $T_{\mathrm{A}}^{*}$ at each position, and then, in Figs. $3 \mathrm{~b}$ and $3 \mathrm{~d}$, contour maps of the integrated $\mathrm{CO}$ intensity, $I(\mathrm{CO})=\int T_{\mathrm{A}}^{*} \mathrm{~d} v$. The integration was over the full range over which significant CO emission is found. For the Central region this was 205 to 255 $\mathrm{km} \mathrm{s}^{-1}$, and for the Southern region this was 205 to 270 $\mathrm{km} \mathrm{s}^{-1}$. The range for the Southern region is larger because there is an additional cloud at $\sim 265 \mathrm{~km} \mathrm{~s}^{-1}$. Most of the emission is between 205 and $245 \mathrm{~km} \mathrm{~s}^{-1}$. For the 


\section{CENTRAL REGION}
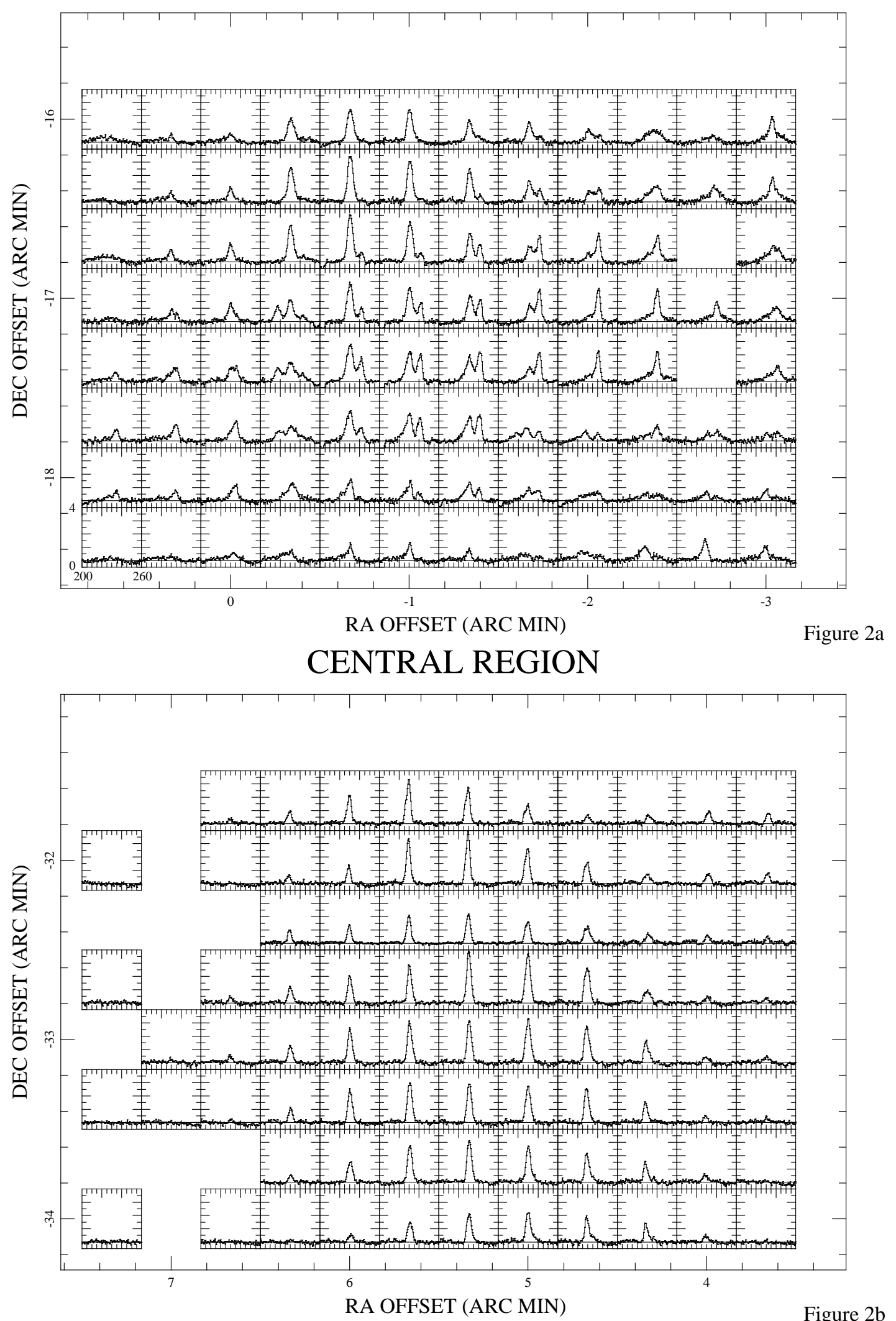

Figure $2 b$

Fig. 2. A selection of spectra from four (three in the Central part and one in the Southern part) different parts of the region. In each region we show a rectangular arrangement of spectra. Boxes are on a half beamwidth grid. For each box the velocity and temperature axes are the same, and are indicated in the sample box in Fig. 2a. $(\alpha, \delta)$ offsets are from the reference position, $\alpha(1950)=5^{\mathrm{h}} 40^{\mathrm{m}} 30^{\mathrm{s}}, \delta(1950)=-69^{\circ} 52^{\prime} 00^{\prime \prime}$ 


\section{CENTRAL REGION}
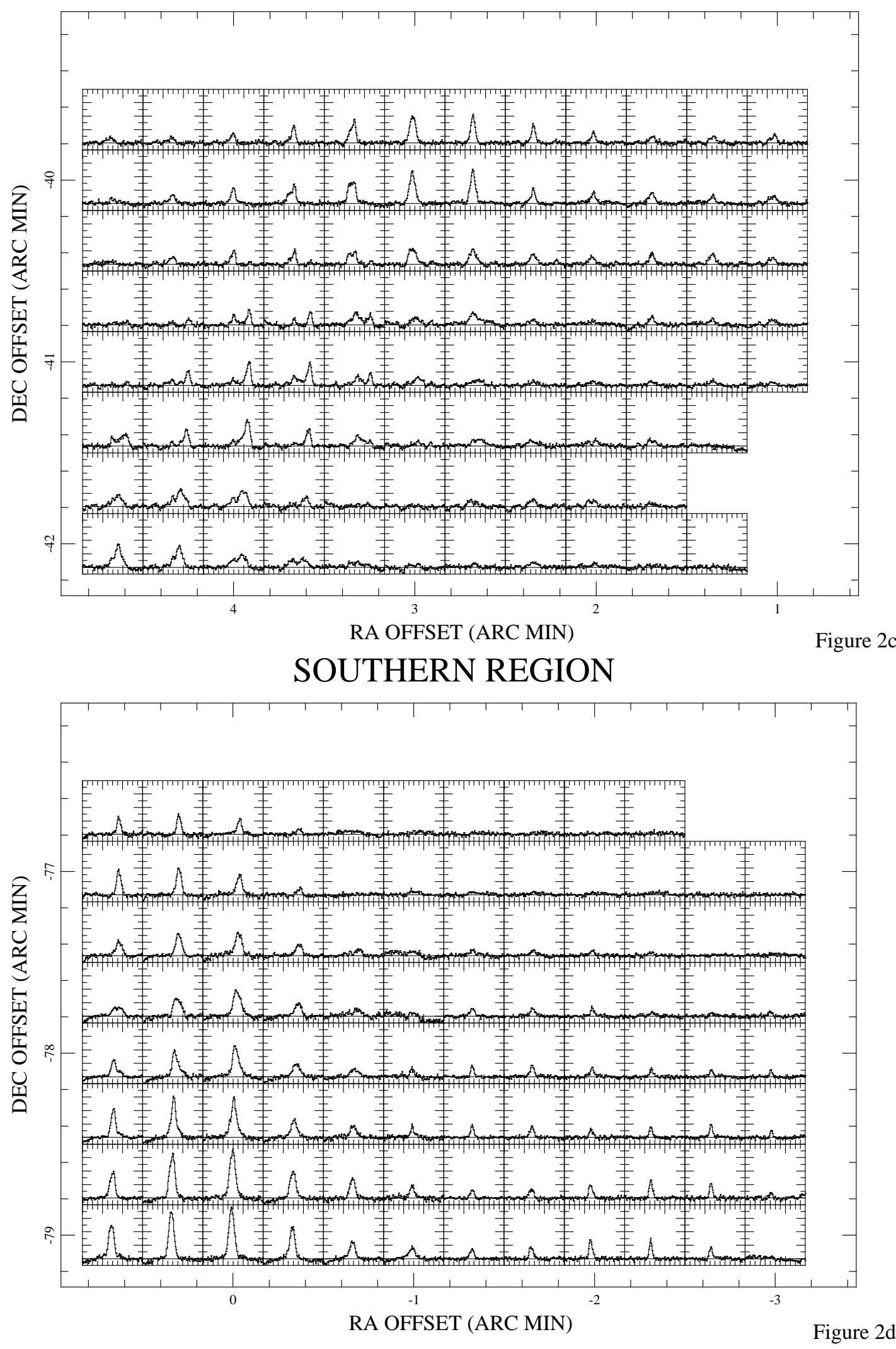

Fig. 2. continued 


\section{CENTRAL REGION - PEAK $\mathrm{T}_{\mathrm{A}}{ }^{*}$}

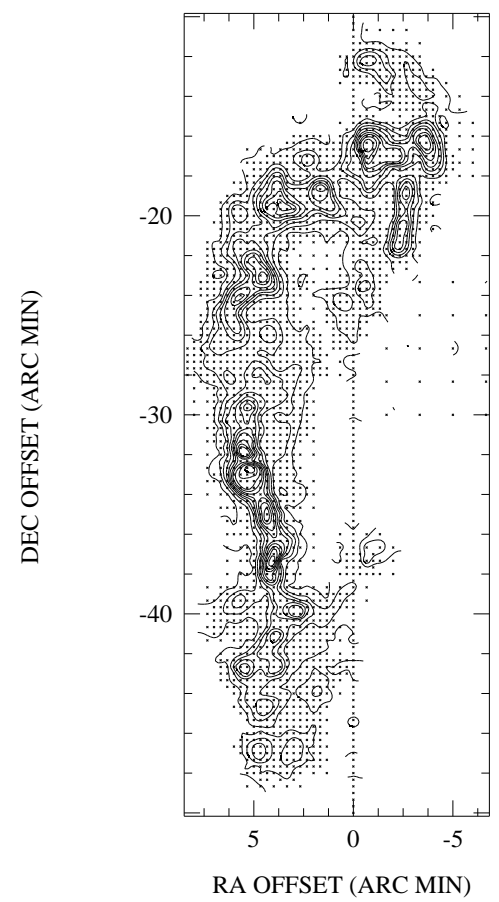

CENTRAL REGION - I(CO)

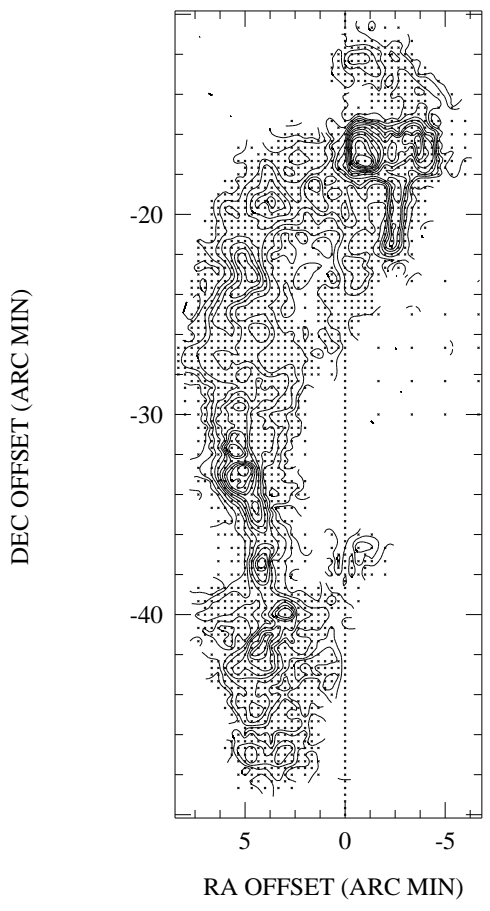

SOUTHERN REGION - PEAK $\mathrm{T}_{\mathrm{A}}{ }^{*}$

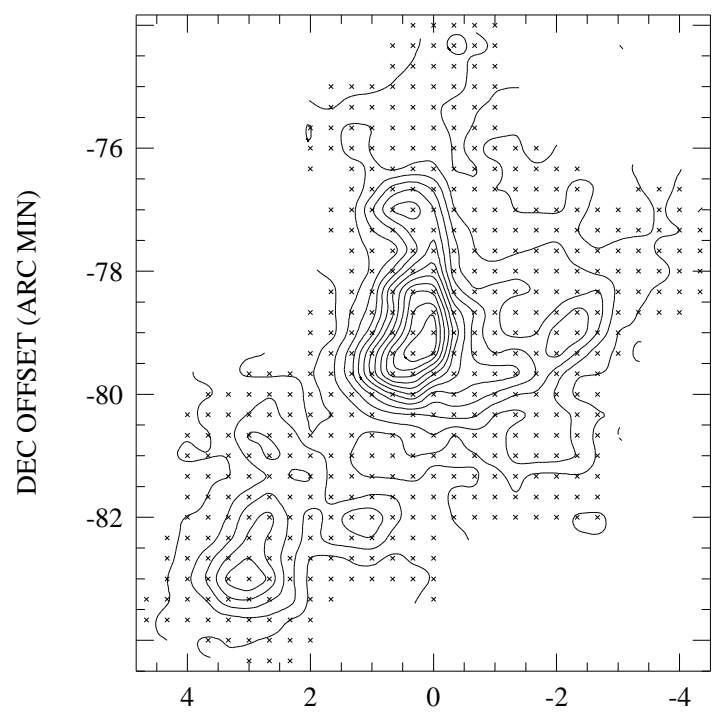

RA OFFSET (ARC MIN)

Figure 3a SOUTHERN REGION - I(CO)

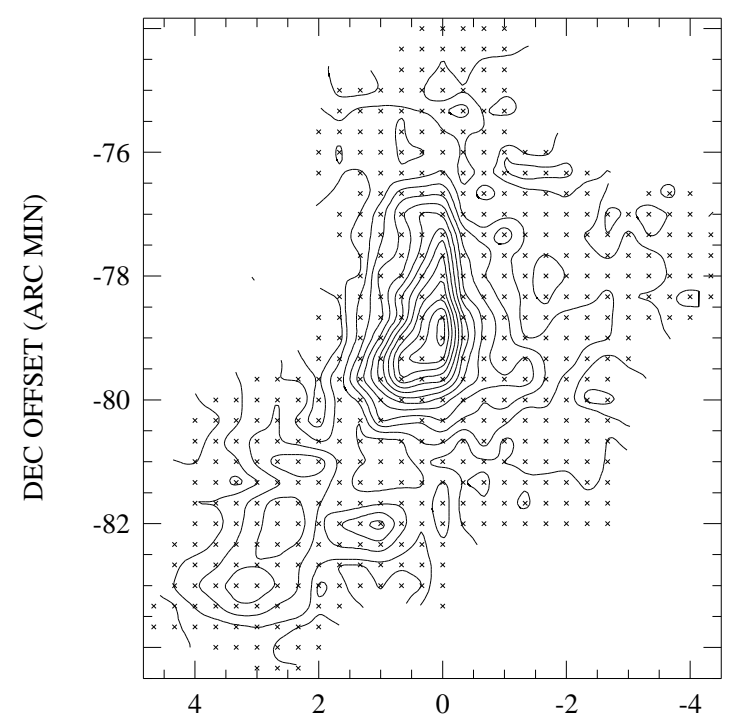

RA OFFSET (ARC MIN)

Figure $3 b$
Figure $3 c$

Fig. 3. CO maps for the Central and Southern regions. In each map, the dots show the locations of observations. $(\alpha, \delta)$ offsets are from the reference position, $\alpha(1950)=5^{\mathrm{h}} 40^{\mathrm{m}} 30^{\mathrm{s}}, \delta(1950)=-69^{\circ} 00^{\prime} 00^{\prime \prime}$. a) Peak $T_{\mathrm{A}}^{*}$ for the Central region. Contour levels are 0.3 to 3.9 in steps of 0.3 (where 0.3 is 3 times the rms noise level). b) Peak $T_{\mathrm{A}}^{*}$ for the Southern region. Contour levels are $0.3 \mathrm{~K}$ to $3.9 \mathrm{~K}$ in steps of $0.3 \mathrm{~K}$ (where $0.3 \mathrm{~K}$ is 3 times the rms noise level). c) $I(\mathrm{CO})$ for the Central region. Contour levels are $1.0,2.0,4.0,6.0, \ldots, 28.0 \mathrm{~K} \mathrm{~km} \mathrm{~s}^{-1}$. The velocity range for the integration is 205 to $255 \mathrm{~km} \mathrm{~s}^{-1}$. d) $I(\mathrm{CO})$ for the Southern region. Contour levels are $1.0,2.0,4.0,6.0, \ldots, 28.0 \mathrm{~K} \mathrm{~km} \mathrm{~s}^{-1}$. The velocity range for the integration is 205 to $270 \mathrm{~km} \mathrm{~s}^{-1}$ 
peak $T_{\mathrm{A}}^{*}$, we chose the contour levels to be in steps of 3 times the rms noise level. Therefore, virtually all features that show up on these maps are real rather than being noise fluctuations. In each of these maps, a dot indicates each of the observed positions.

In the Central region, we see a striking extended feature. It has the appearance of being part of an arc, and is some $600 \mathrm{pc}$ in extent. Even in the integrated or peak intensity maps, it breaks into a large number of CO concentrations. This type of structure is similar to that seen in rich GMC complexes in the Milky Way, e.g the OrionMonoceros complex. In the Southern region, the emission is not as extended, being only $\sim 150 \mathrm{pc}$ in extent. One peak is obvious, and there are other sub-peaks around. This is similar to Milky Way complexes with a few GMCs.

To separate the emission into individual clouds, it is important to isolate emission in individual velocity ranges. In the Milky Way, the typical cloud-cloud velocity dispersion is about 5 to $6 \mathrm{~km} \mathrm{~s}^{-1}$ (Stark 1979), so it is convenient to use bins of approximately that size. This is a convenient range for the LMC also. Therefore, in Fig. 4, we present contours of $I(\mathrm{CO})$ integrated over successive velocity ranges, each $5.0 \mathrm{~km} \mathrm{~s}^{-1}$ wide. In our efforts to isolate individual clouds, we prepared two set of maps offset half of this step $\left(2.5 \mathrm{~km} \mathrm{~s}^{-1}\right)$ from these maps, so we would not miss emission at the edges of the integration ranges. These have not been reproduced here, because the information they contain overlaps with that in Fig. 4. For each region we show maps over the velocity range for which significant emission is seen. Note that for the Southern region there is no emission between 240 and $255 \mathrm{~km} \mathrm{~s}^{-1}$.

In the Central region, the channel maps show that the emission is coming from clouds that are localized in position and velocity. This is important since it shows that it is meaningful to talk about the $\mathrm{CO}$ emission as coming from clouds (possibly like those in the Milky Way), rather than having some extended uniform emission. In the Southern region, we see the emission breaking into cloud like structures. These channel maps have been used to identify individual molecular clouds. Some 22 clouds were found in the Central region and some 5 clouds were found in the Southern region. The identification of these clouds, and their large scale properties (sizes, velocity dispersions, CO luminosities and masses), will be discussed in Paper VII.

The basic observed properties of the clouds that we have identified are shown in Table 1 . In Col. 1 we give the cloud name, in Cols. 2 and 3 , we give the $(\alpha, \delta)$ offsets from the reference position $\left[\alpha(1950)=5^{\mathrm{h}} 40^{\mathrm{m}} 30^{\mathrm{s}}\right.$, $\left.\delta(1950)=-69^{\circ} 52^{\prime} 00^{\prime \prime}\right]$. In Cols. (4) and (5) we give the coordinates of the peak. In Col. (6) we give the peak $T_{\mathrm{MB}}$, and in Col. (7) we give $I(\mathrm{CO})$ at the peak. In Col. (8), is the lsr velocity of the peak, in Col. (9), we give the linewidth at the peak, expressed as a dispersion, $\sigma_{\mathrm{rv}}$. These are the formal temperature weighted dispersions (rather than simply being the result of fitting). If the line were a gaussian, then the full width a half maximum would be $2.35 \sigma_{\mathrm{rv}}$.

The short names given in Table 1 recognize the division of the clouds into the Central and Southern complexes. Within those complexes the clouds are ordered, roughly, in order of their distance from 30Dor. Thus clouds with numbers near each other, also appear near each other in the sky. In addition to the short names given in Table 1, we have also assigned formal names to the clouds, according to the convention proposed by the IAU, as described by Dickel et al. (1987). These formal designations are also given in Table 1.

In order to look for systematic velocity structure, such as rotation or expansion, it is useful to look at coordinatevelocity maps. A selection of these maps is shown in Fig. 5. In Fig. 5a we see declination-velocity plots for the Central region. The first is at an RA offset of $-2.33^{\prime}$. This allows us to investigate the velocity structure in the western extension of the cloud. There is no strong pattern. Note that the emission that protrudes south from the northwest corner of the arc, mostly associated with cloud 30Dor Central 04 , is at a $v_{\text {lsr }}$ of about $240 \mathrm{~km} \mathrm{~s}^{-1}$, while the rest the emission from the northern part of the arc is at $225 \mathrm{~km} \mathrm{~s}^{-1}$. This suggests that the protruding cloud is kinematically distinct from the rest of the arc. The next $\delta-v$ plot is at an RA offset of $+4^{\prime}$, which brings it through the longest part of the cloud. There is very little velocity structure at the top and bottom, except for the presence of a second source at DEC offset $-41^{\prime}$, at $245 \mathrm{~km} \mathrm{~s}^{-1}$. In the center of the main part of the emission, there is a trend of higher velocities as one goes farther north (the same sense as the gradient in the Southern region). This shows up more clearly in the next frame, which shows a $\delta-v$ plot at RA offset of $+5^{\prime}$, where the emission from the Central region is stronger. The gradient along there is $0.9 \mathrm{~km} \mathrm{~s}^{-1}$ $\operatorname{arcmin}^{-1}$ or $0.06 \mathrm{~km} \mathrm{~s}^{-1} \mathrm{pc}^{-1}$. We have also looked at a number of right-ascension-velocity plots at various declinations, and no obvious patterns are seen. These are not presented here.

In Fig. 5b we present coordinate-velocity plots for the Southern region. The lower panel, which shows a $\delta-v$ diagram, exhibits two clear peaks, one at $228 \mathrm{~km} \mathrm{~s}^{-1}$, and the other at $236 \mathrm{~km} \mathrm{~s}^{-1}$. In comparing this with the $I(\mathrm{CO})$ map for the same region, only the stronger peak is visible, while the weaker one is simply lost in the broader emission. With the coordinate velocity map, we can see that these are two distinct peaks. The smooth connection between the two could result from the overlap of the emission from two distinct clouds, or it could be a real connection, with the velocity shift arising from cloud rotation, with the upper part of the complex moving away from us. This would correspond to a velocity gradient of $3.5 \mathrm{~km} \mathrm{~s}^{-1} \operatorname{arcmin}^{-1}$, or $0.2 \mathrm{~km} \mathrm{~s}^{-1} \mathrm{pc}^{-1}$. In the upper frame, showing an $\alpha-v$ diagram, we again see two distinct peaks, but only a small velocity shift or gradient. 
Table 1. Parameters of CO peaks in 30DOR Complex

\begin{tabular}{|c|c|c|c|c|c|c|c|c|c|c|c|c|c|c|c|c|}
\hline \multirow[t]{2}{*}{ Short name } & \multicolumn{5}{|c|}{ Offsets $^{1}$} & \multicolumn{6}{|c|}{ Coordinates } & \multicolumn{4}{|c|}{ CO line parameters } & \multirow[t]{2}{*}{ Long name ${ }^{2}$} \\
\hline & $\Delta a c$ & $\cos \delta$ & & $\Delta \delta$ & & & 1950 & & & 1950 & & $\begin{array}{l}T_{\mathrm{mb}} \\
(\mathrm{K})\end{array}$ & $\begin{array}{c}I(\mathrm{CO}) \\
\left(\mathrm{K} \mathrm{km} \mathrm{s}^{-1}\right)\end{array}$ & $\begin{array}{c}V_{\mathrm{LSR}} \\
\left(\mathrm{km} \mathrm{s}^{-1}\right)\end{array}$ & $\begin{array}{c}\sigma_{\mathrm{RV}} \\
\left(\mathrm{km} \mathrm{s}^{-1}\right)\end{array}$ & \\
\hline 30DOR-CENTER-01 & $-00^{\prime}$ & $40^{\prime \prime}$ & $.0^{\circ}$ & $12^{\prime}$ & $20^{\prime \prime}$ & & $40^{m_{2}}$ & $22^{8}$ & $-70^{\circ}$ & $04^{\prime} 2$ & $20^{\prime \prime}$ & $2.22 \pm 0.11$ & $15.8 \pm 0.17$ & 230 & 4.14 & LMC:CO B054022-700420 VLSR=230 SEST \\
\hline 30DOR-CENTER-02 & -03 & 20 & -0 & 16 & 20 & $\mathbf{5}$ & 39 & $\mathbf{5 0}$ & -70 & 08 & 20 & $3.82 \pm 0.14$ & $23.1 \pm 0.20$ & 238 & 2.94 & LMC:CO B053950-700820 VLSR $=238$ SEST \\
\hline 30DOR-GENTER-03 & -00 & 40 & -0 & 16 & 40 & 5 & 40 & 22 & -70 & 08 & 40 & $4.60 \pm 0.09$ & $33.8 \pm 0.13$ & 230 & 3.22 & LMC:CO B054022-700840 VLSR=230 SEST \\
\hline 30DOR-CENTER-04 & -02 & 20 & -02 & 21 & 40 & 5 & 40 & 02 & -70 & 13 & 40 & $2.92 \pm 0.11$ & $20.4 \pm 0.15$ & 226 & 2.89 & LMC:CO B054002-701340 VLSR $=226$ SEST \\
\hline 30DOR-CENTER-05A & -00 & 20 & -02 & 23 & 40 & 5 & 40 & 26 & -70 & 15 & 40 & $1.73 \pm 0.11$ & $9.3 \pm 0.13$ & 215 & 2.68 & LMC:CO B054026-701540 VLSR=215 SEST \\
\hline 30DOR-CENTER-05B & -01 & 00 & $-0:$ & 23 & 00 & 5 & 40 & 18 & -70 & 15 & 00 & $0.63 \pm 0.10$ & $3.6 \pm 0.13$ & 222 & 1.93 & LMC:CO B054018-701500 VLSR $=222$ SEST \\
\hline 30DOR-CENTER-05C & -00 & 20 & -0 & 22 & 40 & 5 & 40 & 26 & -70 & 14 & 40 & $1.26 \pm 0.12$ & $7.9 \pm 0.15$ & 229 & 3.07 & LMC:CO B054026-701440 VLSR $=229$ SEST \\
\hline 30DOR-CENTER-06 & 03 & 40 & -0 & 19 & 40 & 5 & 41 & 14 & -70 & 11 & 40 & $3.68 \pm 0.12$ & $17.8 \pm 0.16$ & 227 & 2.23 & LMC:CO B054114-701140 VLSR $=227$ SEST \\
\hline 30DOR-CENTER-07 & 04 & 40 & -0 & 23 & 20 & 5 & 41 & 25 & -70 & 15 & 20 & $3.51 \pm 0.13$ & $19.1 \pm 0.24$ & 226 & 3.51 & LMC:CO B054125-701520 VLSR $=226$ SEST \\
\hline 30DOR-CENTER-08 & 04 & 20 & -0 & 26 & 00 & 5 & 41 & 21 & -70 & 18 & 00 & $1.79 \pm 0.12$ & $11.8 \pm 0.17$ & 229 & 3.41 & LMC:CO B054121-701800 VLSR $=229$ SEST \\
\hline 30DOR-CENTER-09 & 06 & 40 & -0 & 26 & 20 & $\mathbf{5}$ & 41 & 49 & -70 & 18 & 20 & $1.96 \pm 0.09$ & $7.8 \pm 0.09$ & 225 & 1.84 & LMC:CO B054149-701820 VLSR=225 SEST \\
\hline 30DOR-CENTER-10 & 05 & 20 & -0 & 29 & 40 & 5 & 41 & 33 & -70 & 21 & 40 & $3.32 \pm 0.11$ & $19.0 \pm 0.15$ & 229 & 2.47 & LMC:CO B054133-702140 VLSR $=229$ SEST \\
\hline 30DOR-CENTER-11 & 05 & 20 & -0 & 32 & 00 & $\mathbf{5}$ & 41 & 33 & -70 & 24 & 00 & $5.19 \pm 0.12$ & $26.0 \pm 0.16$ & 230 & 2.14 & LMC:CO B054133-702400 VLSR $=230$ SEST \\
\hline 30DOR-CENTER-12 & 04 & 00 & -0 & 37 & 20 & $\mathbf{5}$ & 41 & 17 & -70 & 29 & 20 & $4.77 \pm 0.10$ & $21.2 \pm 0.13$ & 229 & 2.25 & LMC:CO B054117-702920 VLSR=229 SEST \\
\hline 30DOR-CENTER-13 & 05 & 40 & -0 & 39 & 20 & 5 & 41 & 37 & -70 & 31 & 20 & $1.66 \pm 0.08$ & $7.8 \pm 0.10$ & 229 & 2.50 & LMC:CO B054137-703120 VLSR $=229$ SEST \\
\hline 30DOR-CENTER-14 & 02 & 40 & -0 & 40 & 00 & 5 & 41 & 02 . & -70 & 32 & 00 & $3.31 \pm 0.13$ & $20.3 \pm 0.18$ & 227 & 2.64 & LMC:CO B054102-703200 VLSR=227 SEST \\
\hline 30DOR-CENTER-15A & 04 & 00 & -0 & 42 & 40 & $\mathbf{5}$ & 41 & 17 & -70 & 34 & 40 & $2.38 \pm 0.09$ & $12.5 \pm 0.10$ & 228 & 2.44 & LMC:CO B054117-703440 VLSR $=228$ SEST \\
\hline 30DOR-CENTER-15B & 05 & 20 & -0 & 42 & 40 & 5 & 41 & 33 & -70 & 34 & 40 & $2.35 \pm 0.13$ & $10.5 \pm 0.14$ & 234 & 2.24 & LMC:CO B054133-703440 VLSR=234 SEST \\
\hline 30DOR-CENTER-15C & 04 & 00 & -0 & 41 & 20 & 5 & 41 & 17 & -70 & $\mathbf{3 3}$ & 20 & $2.69 \pm 0.11$ & $17.3 \pm 0.12$ & 244 & 2.34 & LMC:CO B054117-703320 VLSR $=244$ SEST \\
\hline 30DOR-CENTER-16 & 04 & 20 & -0 & 44 & 40 & 5 & 41 & 21 & -70 & 36 & 40 & $1.87 \pm 0.10$ & $7.9 \pm 0.12$ & 230 & 2.03 & LMC:CO B054121-703640 VLSR $=230$ SEST \\
\hline 30DOR-CENTER-17 & 02 & 00 & -0 & 44 & 00 & 5 & 40 & 54 & -70 & 36 & 00 & $0.98 \pm 0.13$ & $6.3 \pm 0.18$ & 238 & 3.55 & LMC:CO B054054-703600 VLSR=238 SEST \\
\hline 30DOR-CENTER-18 & 04 & 40 & -0 & 46 & 40 & 5 & 41 & 25 & -70 & 38 & 40 & $1.78 \pm 0.11$ & $13.4 \pm 0.18$ & 235 & 4.28 & LMC:CO B054125-703840 VLSR=235 SEST \\
\hline 30DOR-SOUTH-01 & 01 & 20 & -1 & 16 & 00 & 5 & 40 & 47 & -71 & 08 & 00 & $1.02 \pm 0.09$ & $7.4 \pm 0.15$ & 260 & 4.69 & LMC:CO B054047-710800 VLSR $=260$ SEST \\
\hline 30DOR-SOUTH-02 & 00 & 20 & -1 & 17 & 00 & 5 & 40 & 34 & -71 & 09 & 00 & $2.61 \pm 0.11$ & $16.1 \pm 0.18$ & 236 & 2.88 & LMC:CO B054034-710900 VLSR $=236$ SEST \\
\hline 30DOR-SOUTH-03 & 00 & 20 & -1 & 19 & 20 & 5 & 40 & 34 & -71 & 11 & 20 & $5.23 \pm 0.15$ & $33.9 \pm 0.21$ & 227 & 2.89 & LMC:CO B054034-711120 VLSR $=227$ SEST \\
\hline 30DOR-SOUTH-04 & 01 & 00 & -1 & 22 & 00 & 5 & 40 & 42 & -71 & 14 & 00 & $1.42 \pm 0.12$ & $9.3 \pm 0.14$ & 228 & 2.46 & LMC:CO B054042-711400 VLSR $=228$ SEST \\
\hline 30DOR-SOUTH-05 & 03 & 00 & -1 & 23 & 00 & 5 & 41 & 07 & -71 & 15 & 00 & $2.44 \pm 0.10$ & $8.1 \pm 0.10$ & 218 & 1.41 & LMC:CO B054107-711500 VLSR $=218$ SEST \\
\hline
\end{tabular}

${ }^{1}$ offsets are from the reference position, $\alpha(1950)=5^{\mathrm{h}} 40^{\mathrm{m}} 30^{\mathrm{s}}, \delta(1950)=-69^{\circ} 52^{\prime} 00^{\prime \prime}$.

${ }^{2}$ Formal designation according to the format described by Dickel et al. (1987).

\section{Discussion}

The most obvious result is that the $\mathrm{CO}$ emission in the 30Dor Complex comes from well defined clouds. The sizes of the clouds shown in this study are in the tens of parsecs. Maps of the spectra have many characteristics of similar maps in Milky Way molecular clouds (except for the line strengths). All of this suggests that is reasonable to talk about the molecular cloud population of the LMC and to compare it with that in the Milky Way. Moreover, the line profiles result from a complex interplay of a variety of cloud conditions, including internal cloud kinematics, as well as temperature, density and abundance variations along the line of sight. The fact that the LMC line profiles appear similar to those in Milky Way molecular clouds suggests that many of these physical conditions are comparable in the LMC and the Milky Way.

Before discussing the individual cloud properties, we first look at the arrangement of clouds in each region. In the Central region, the clouds are arranged in an elongated structure, approximately $600 \mathrm{pc}$ long. Optical images show that this coincides with a dust lane. The IRAS point sources in this region follow along the peak of the CO emission. In addition Caldwell \& Kutner (1992) and Caldwell et al. (1993) have shown that there is extended FIR emission associated with this structure.

The clouds in this structure appear close together. In fact there is so much overlap that the individual clouds are only apparent in the maps covering restricted velocity ranges. This compact arrangement is in contrast to that of the N11 region, in which there are very clear gaps between most of the clouds (Johansson 1991; de Graauw et al. 1996). This difference in arrangement suggests that the local physical conditions may be very different in the complex south of 30Dor and in the N11 region.

The emission in the Southern region appears to be separate from that in the Central region. (If there is a connection between the two regions, it would probably be about 4 minutes east of the fully sampled N-S strip. That strip, does show some weak emission between the two regions, but we have not yet followed that up). The Southern region appears to have a much simpler structure than the Central region. The clouds there still have some overlap. There is a single strong FIR source at the peak of the $\mathrm{CO}$ emission. In addition, there is extended FIR emission covering the whole area in which CO emission is seen (Caldwell \& Kutner 1992, 1996; Caldwell et al. 1993).

\subsection{Comparison with Milky Way line temperatures}

We have noted that, while the line profiles appear similar to those in Milky Way molecular clouds, the LMC lines are systematically weaker. We first ask whether this is could result from the effects of beam dilution at the greater distances of the LMC clouds. That is, if we took a typical Milky Way cloud complex and moved it to a distance of $50 \mathrm{kpc}$, how strong would the emission appear?

To answer this question, we can look at fully sampled maps of the Orion region, and degrade the resolution to 10 pc. In the undegraded $\mathrm{CO}(1 \rightarrow 0)$ maps (with $0.1 \mathrm{pc}$ 
CENTRAL REGION

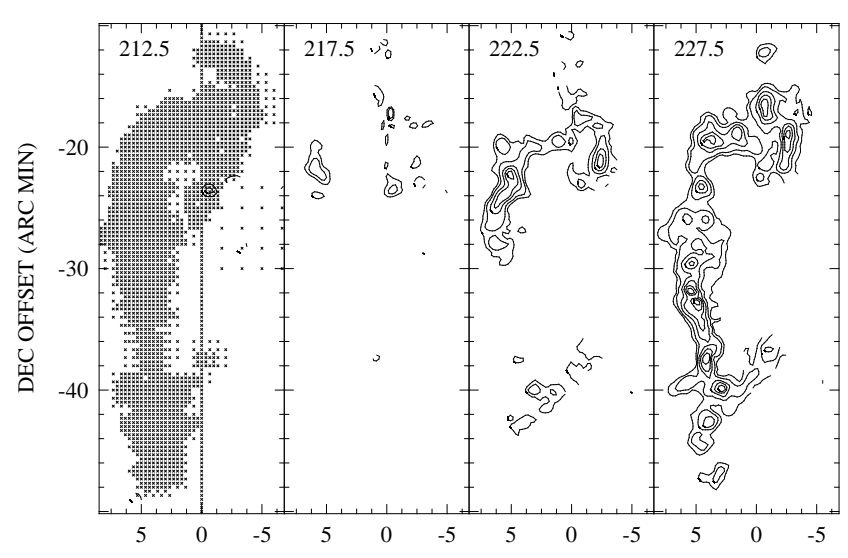

RA OFFSET (ARC MIN)

\section{CENTRAL REGION}

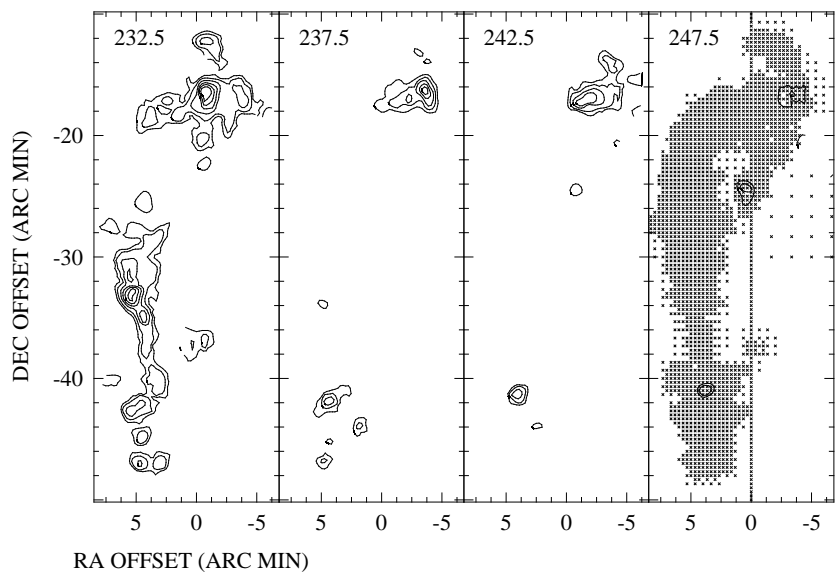

SOUTHERN REGION

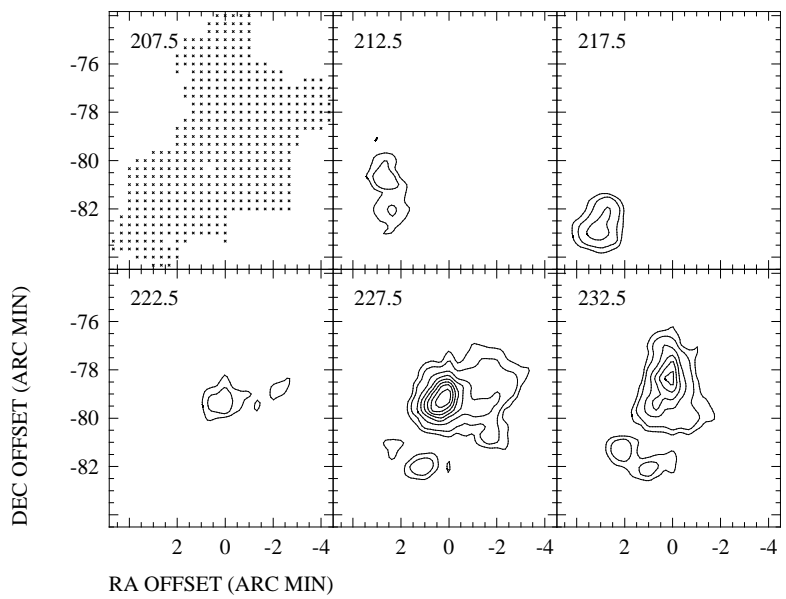

SOUTHERN REGION

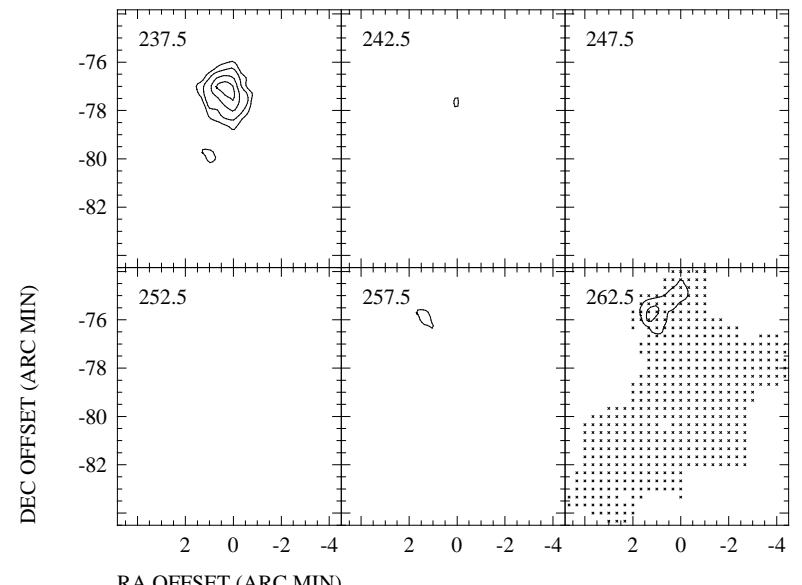

Fig. 4. CO channel maps the Central and Southern regions. The maps are integrated in $5 \mathrm{~km} \mathrm{~s}^{-1}$ steps covering the full range over which significant emission is seen. The central velocity for the $5 \mathrm{~km} \mathrm{~s}^{-1}$ range is shown on each panel. For each region, the dots showing the locations of the observations are in the first and last maps only. Contours are 1.0, 2.0, 4.0, 6.0, ..., $14.0 \mathrm{~K} \mathrm{~km} \mathrm{~s}^{-1}$ for all of the maps. $(\alpha, \delta)$ offsets are from the reference position, $\alpha(1950)=5^{\mathrm{h}} 40^{\mathrm{m}} 30^{\mathrm{s}}, \delta(1950)=-69^{\circ} 52^{\prime} 00^{\prime \prime}$

linear resolution), $T_{\mathrm{MB}}$ at the peak is about $75 \mathrm{~K}$, and in the extended cloud envelopes is about $5 \mathrm{~K}$. In the degraded maps, the $T_{\mathrm{MB}}$ at the peak is about $20 \mathrm{~K}$, and in the envelopes is about $4 \mathrm{~K}$. (Note, the peak only falls by a factor of $\sim 4$ because there is still strong emission $5 \mathrm{pc}$ from the peak, and the envelopes do not change by even that much, because there is little variation in intensity on the $5 \mathrm{pc}$ scale). In the LMC, the strongest peaks in the 30 Dor Central and South regions (Table 1) are 5.2 K. These are a factor of $\sim 4$ weaker than in the degraded Milky Way maps. In the extended envelopes (not near the peaks or too close to the cloud edges), the lines are 1 to $2 \mathrm{~K}$. Again, these average a factor of $\sim 4$ weaker than in the degraded Milky Way maps.

It therefore appears that the difference between the LMC and Milky Way line strengths is a real one. This means that there are some physical conditions that affect line strength, and are different in the LMC and the Milky Way. The most obvious of these are:

1. Abundances. The lower metallicity of the LMC results in lower $[\mathrm{C}] /[\mathrm{H}]$ and $[\mathrm{O}] /[\mathrm{H}]$. We would therefore expect a lower $[\mathrm{CO}] /\left[\mathrm{H}_{2}\right]$. This lower abundance would produce lower $\mathrm{CO}$ column densities and therefore weaker $\mathrm{CO}$ lines. One might think that, since the ${ }^{12} \mathrm{CO}(1 \rightarrow 0)$ line is optically thick in most Milky Way molecular clouds, then dropping the column density should not have a significant effect on line strength. However, we know that trapping plays an important role in $\mathrm{CO}$ excitation and reducing the column density can reduce the excitation temperature. This effect is most pronounced in the lower density parts of the cloud where the line is less easily thermalized. 


\section{CENTRAL REGION}
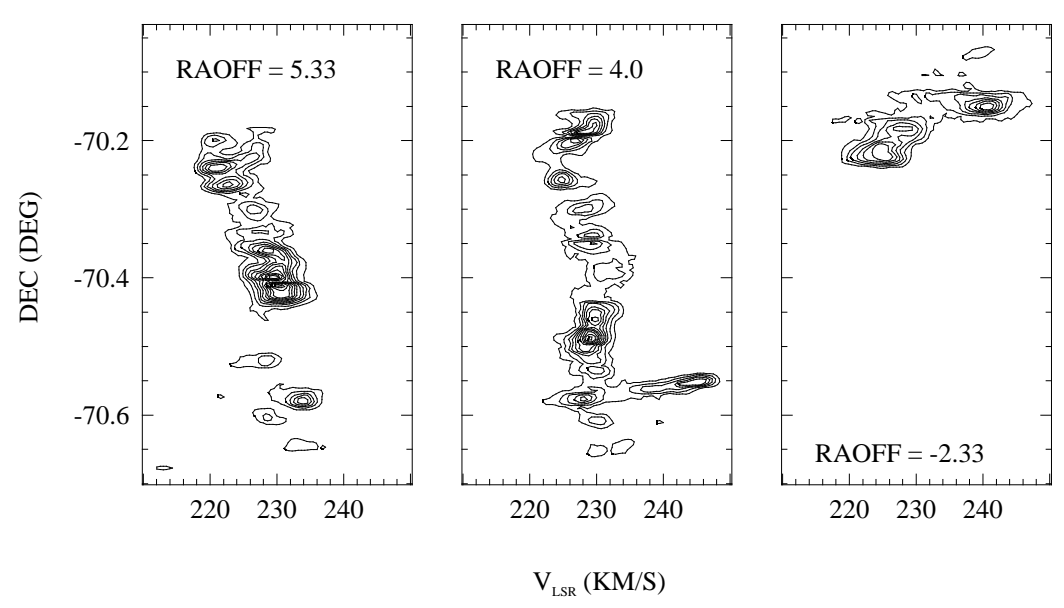

SOUTHERN REGION

Figure 5a
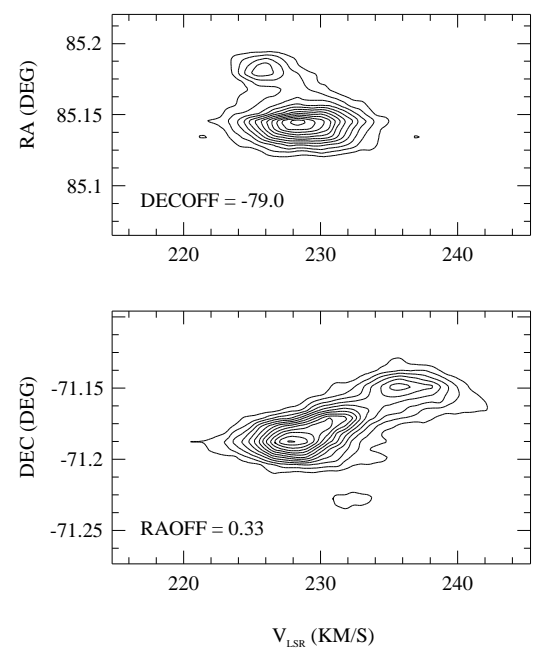

Figure $5 b$

Fig. 5. Selected coordinate velocity maps for the Central region and for the Southern region. $(\alpha, \delta)$ offsets are from the reference position, $\alpha(1950)=5^{\mathrm{h}} 40^{\mathrm{m}} 30^{\mathrm{s}}, \delta(1950)=-69^{\circ} 52^{\prime} 00^{\prime \prime}$

2. Excitation. In addition to the trapping effects, mentioned above, it is possible that the temperatures and densities in the cloud are lower than in their Milky Way counterparts. These would result in lower excitation temperatures and weaker lines. Heating at the strongest peaks should be by embedded O stars. Caldwell \& Kutner find that the FIR luminosities of these clouds are comparable to active clouds in the Milky Way, suggesting comparable internal heating. The extended envelopes of the clouds are heated by the interstellar radiation field (ISRF). Evidence suggests that the ISRF is not weaker in the LMC than in the Milky Way.
3. Clumping effects. There is growing evidence in Milky Way cloud cores and extended envelopes, that the CO emission is coming from clumps (on roughly the $0.1 \mathrm{pc}$ scale) that fill a certain fraction of the cloud volume. The temperatures, densities and abundances that we measure from molecular line studies are within the clumps. The velocity dispersions that we measure are often dominated by the clump-clump motions. The properties of the lines that we see depend on the physical conditions in the clumps as well as the filling factor of the clumps. It is possible that in the LMC, the clump properties are different, or that they fill a smaller fraction of the volume than in the Milky Way. 
We present these ideas to explore the range of plausible explanations for the weaker lines in the LMC. Further analysis of these possibilities requires observations of different transitions and different isotopic species, as well as a line formation analysis that takes clumping and trapping into account. Some discussion of these issues for the SMC are in Paper IV.

\subsection{Comparison with $1.2 \mathrm{~m}$ data}

The Central region is associated with clouds \#35 and \#36 identified with the large scale CO survey of the LMC by Cohen et al. (1988), made with a $1.2 \mathrm{~m}$ radio telescope (FWHP beam of 8.8'). The Southern region lies projected toward cloud \#34 in Cohen et al. It is interesting to determine the degree to which clouds we find with high angular resolution account for the CO luminosity seen by the $1.2 \mathrm{~m}$ telescope. If there is significant extended low level emission beyond the boundaries of the clouds that we have mapped with the SEST, then we would expect the total luminosity of the $1.2 \mathrm{~m}$ telescope to be larger than the sum of the SEST luminosities. That is because the larger beam includes the luminosities of the clouds and the extended component. If there is no significant extended component, then we would expect both telescopes to measure the same total luminosity.

In order to compare the low and high resolution luminosities, we have added all the spectra taken with SEST toward both the Central and Southern regions and those taken with the $1.2 \mathrm{~m}$ dish at CTIO covering the same areas. In Table 2, we summarize the parameters obtained from a single gaussian fit to the integrated spectra. As can be seen in Col. 5, the luminosities derived from the SEST and CTIO dishes are in good agreement, suggesting that essentially all the $\mathrm{CO}$ emission detected with the low angular resolution is coming from the clouds mapped with the SEST. In fact, the SEST luminosities are about $40 \%$ larger than those observed with the $1.2 \mathrm{~m}$ telescope, making very unlikely the presence of extended low level component of the $\mathrm{CO}$ emission. The lower values of the luminosities observed with the $1.2 \mathrm{~m}$ dish are probably due to beam efficiency effects, since the arc like structure generally runs off the main beam central position.

Table 2. Luminosity comparison

\begin{tabular}{ccccc}
\hline \hline Region & Telescope & $\begin{array}{c}V_{\mathrm{LSR}} \\
\left(\mathrm{km} \mathrm{s}^{-1}\right)\end{array}$ & $\begin{array}{c}\Delta v \\
\left(\mathrm{~km} \mathrm{~s}^{-1}\right)\end{array}$ & $\begin{array}{c}L_{\mathrm{CO}} \\
\left(\mathrm{K} \mathrm{km} \mathrm{s}^{-1}\right)\end{array}$ \\
\hline Central & SEST & $228.6 \pm 0.7$ & $10.5 \pm 1.8$ & $3.310^{5}$ \\
& CTIO & $228.9 \pm 0.8$ & $9.2 \pm 1.7$ & $2.210^{5}$ \\
Southern & SEST & $228.5 \pm 0.9$ & $5.4 \pm 1.5$ & $2.810^{4}$ \\
& CTIO & $227.7 \pm 1.1$ & $6.8 \pm 2.8$ & $2.210^{4}$ \\
\hline
\end{tabular}

\subsection{Comparison with other tracers}

In understanding the evolution of the interstellar medium, and in particular the formation and destruction of the molecular clouds, it is important to examine the relationship between the atomic and the molecular gas. The most sensitive HI maps of this section of the LMC (but with a $15^{\prime}$ beam, and $12^{\prime}$ sampling) are by Rohlfs et al. (1984) and Luks \& Rohlfs (1992). At this resolution, we can only look for general trends relative to our data. (We would hope, now that the Australia Telescope is available that $\mathrm{HI}$ maps with resolution comparable to our $\mathrm{CO}$ maps will soon be available).

In comparing the HI maps (especially the "disk" component identified by Luks \& Rholfs) with the CO maps of Cohen et al., we see that for the whole 30 Dor Complex, the HI generally follows the $\mathrm{CO}$. The $\mathrm{HI}$ is generally more extended, but the strongest emission is generally in the same place. The Central region corresponds with a distinct peak in the HI. The Southern region is part of an extended HI sub-peak.

In comparing velocity components, for the Central region, the HI has two components. The higher velocity component is at $v_{\mathrm{lsr}}$ ranging from 253 to $275 \mathrm{~km} \mathrm{~s}^{-1}$. There is no $\mathrm{CO}$ emission from the Central region in this velocity range. However, there is a lower velocity HI component, which very closely follows the $\mathrm{CO}$ velocity in the Central region. It even shows the shifts with position evidenced in the CO (e.g. Figs. 5c, d). In the direction of the Southern region, the HI also has two components. The higher component is at $261 \mathrm{~km} \mathrm{~s}^{-1}$, and there is one CO cloud at approximately that velocity. The lower velocity component is at $234 \mathrm{~km} \mathrm{~s}^{-1}$, which is the same as that of most of the $\mathrm{CO}$ emission in this region (e.g. Figs. 5a, b).

We also note other features found in the two regions. The Central region has six IRAS point sources falling within the boundaries of the CO emission. The $100 \mu \mathrm{m}$ flux densities range from 26 to $44 \mathrm{Jy}$. Each of the sources appears close to a CO peak. The Southern region has one IRAS point source, with a $100 \mu \mathrm{m}$ flux density of $112 \mathrm{Jy}$. It appears located at the peak of the $\mathrm{CO}$ emission. We should note that, in between the two regions, east of the N-S strip, there are two IRAS point sources. This region has not yet been surveyed by SEST.

\section{Summary}

We present fully sampled maps of ${ }^{12} \mathrm{CO}(1 \rightarrow 0)$ emission from a section of the Large Magellanic Cloud, made with the SEST. At the distance of the LMC, the $43^{\prime \prime}$ angular resolution of the SEST corresponds to $10 \mathrm{pc}$. The region studied is part of the molecular cloud complex that extends $2 \mathrm{kpc}$ south of 30Dor. The observations presented here are of the Central and Southern parts of that complex. 
The appearance of the spectra, and their variation with position, is very much like those seen in Milky Way giant molecular clouds. The emission also comes from well defined molecular clouds, rather than from a low level diffuse emission. The most significant difference is that the LMC lines are weaker, with the strongest $T_{\mathrm{MB}}=5.2 \mathrm{~K}$ and the largest $I(\mathrm{CO})=33.9 \mathrm{~K} \mathrm{~km} \mathrm{~s}^{-1}$. Line temperatures both at the peaks and in the cloud envelopes are a factor of $\sim 3$ to 5 weaker than those found for Milky Way GMCs even when the resolution of the Milky Way observations is degraded to $10 \mathrm{pc}$.

In the Central region, the emission comes from an extended feature that has the appearance of a section of an arc. The arc is some 600 pc long. Even in peak or $I(\mathrm{CO})$ maps, the arc breaks into several sub structures. There is a definite break between the Central and Southern regions. In the Southern region, the emission is on some $150 \mathrm{pc}$ in extent. Using channel maps in $5 \mathrm{~km} \mathrm{~s}^{-1}$ steps, we have been able to separate the emission in these regions into well defined clouds. In the Central region, there are 22 clouds and in the Southern region there are 5 clouds. The cloud properties (size, velocity dispersion, virial mass and CO luminosity) will be discussed in a future paper.

Acknowledgements. M.L.K. would like to acknowledge travel support from an AAS Small Research Grant, and the NRAO Fund for Travel to Unique Foreign Telescopes, NASA grants NAG 2-677, NAG 5-1631, NAG 5-2302. We would also like to thank Dr. Jean Chiar for assistance in the data reduction. M.R. would like to acknowledge support from FONDECYT(CHILE) through grant \#1930928.

\section{References}

Caldwell D., Kutner M.L., 1996, ApJ 472, 611

Caldwell D., Kutner M.L., 1992, Proc. Heidelberg Conference New Aspects of Magellanic Cloud Research, Klare G. (ed.). Springer-Verlag
Cohen R.S., Dame T.M., Garay G., Montani J., Rubio M., Thaddeus P., 1988, ApJL 331, L95

Combes F., 1991, ARA\&A, 195

de Graauw Th., et al., 1996 (in preparation)

Dickel H.R., Lortet M.-C., de Boer K.S., 1987, A\&AS 68, 75

Dufour, 1984, IAU Symp. 108, Structure and Evolution of the Magellanic Clouds. In: vanden Bergh S. and de Boer K.S. (eds.). Reidel, Dordrecht, p. 353

Israel F.P., Johansson L.E.B., Lequeux J., et al., 1993, A\&A 276, 25 (Paper I)

Johansson L.E.B., 1991, IAU Symposium 146: Dynamics of Galaxies and Their Molecular Cloud Distribuition. In: Combes F. and Casoli F. (eds.). Kluwer, p. 1

Koornneef, 1982, A\&A 107, 247

Kutner M.L., Ulich R.L., 1981, ApJ 250, 341

Lequeux J., Le Bourlot J., Pineau des Forets G., Roueff E., Boulanger F., Rubio M., 1994, A\&A 292, 371 (Paper IV)

Luks Th., Rohlfs K., 1992, A\&A 263, 41

Mead K.N., Kutner M.L., 1988, ApJ 330, 399

Rohlfs K., Kreitschmann J., Siegman B.C., Feitzinger J.V., 1984, A\&A 137, 347

Rubio M., Garay G., Montani J., Thaddeus P., 1991, ApJ 368, 173

Rubio M., 1991, IAU Symposium 146: Dynamics of Galaxies and Their Molecular Cloud Distribuition. In: Combes F. and Casoli F. (eds.). Kluwer, p. 9

Rubio M., Lequeux J., Boulanger F., et al., 1993, A\&A 271, 1 (Paper II)

Rubio M., Lequeux J., Boulanger F., 1993, A\&A 271, 9 (Paper III)

Rubio M., Lequeux J., Boulanger F., et al., 1996, A\&AS 118, 263 (Paper V)

Stark A.A., 1979, Ph. D. Thesis, Princeton University

Young J., Scoville N., 1991, ARA\&A, 581 